\title{
快速反射镜状态模型构建方法及其控制系统设计
}

\author{
艾志伟，嵇建波，李 静，黄书童 \\ (桂林航天工业学院, 广西 桂林 541004)
}

\begin{abstract}
摘要: 为减少快速反射镜状态空间建模过程中所需的结构参数数量, 提出了一种基于系统辨识的状态 模型构建方法, 采用该方法建立状态模型时只需使用音圈电阻和电感两个结构参数。基于状态模型, 设计了一套由降阶观测器、状态反馈、内模和镇定补偿器构成的组合控制系统, 利用状态反馈完成对 内模和镇定补偿器的设计, 通过设计降阶观测器实现对电流和角速度的获取, 组合系统可同时实现对 输入信号的渐进跟踪和干扰的抑制。在 SIMULINK 中建立仿真模型, 仿真结果显示, 不考虑干扰作 用时，相较于不完全微分 PID（Proportion Integration Differentiation）控制系统，组合系统的调节时间 下降了 53.6\%, 超调量上升了 131.2\%; 加入干扰信号后，不完全微分 PID 控制系统的动稳态性能有明 显下降, 而组合系统的输出性能基本不受影响。仿真结果验证了理论分析的正确性。
\end{abstract}

关键词: 快速反射镜; 状态模型; 渐进跟踪; 干扰抑制

中图分类号：TP273 文献标识码：A 文章编号：1001-8891(2020)01-0040-06

\section{State Model Construction Method for Fast Steering Mirror and Its Control System Design}

\author{
AI Zhiwei， JI Jianbo， LI Jing, HUANG Shutong \\ (Guilin University of Aerospace Technology, Guilin 541004, China)
}

\begin{abstract}
To reduce structural parameters needed in the process of the state space modeling of a fast steering mirror (FSM), a modeling method based on system identification is proposed. Only two structural parameters, namely, coil resistance and inductance, are needed when using this modeling method. Based on the state model, a set of combined control systems consisting of a reduced-order observer, state feedback, an internal model, and a stabilization compensator were designed; the internal model and stabilization compensator were designed by using state feedback. Furthermore, current and angular velocity can be obtained using a reduced-order observer. The combined system can progressively track of input signals and suppress disturbances; the simulation model was built using SIMULINK. The simulation results show that, compared with the incomplete differential proportional integral derivative (PID) control system, the setting time decreases by $53.6 \%$ and the overshoot increases by $131.2 \%$ without considering the disturbance in the composite system. The dynamic and steady-state performance of the incomplete differential PID control system decreases significantly after adding the disturbance signal, while the composite system is maintained. The simulation results verify the correctness of the theoretical analysis.
\end{abstract}

Key words: fast steering mirror, state model, progressive tracking, disturbance suppression

\section{0 引言}

快速反射镜是一种精密调整光束传播方向的装 置, 具有结构紧凑、调整精度高、调节速度快的优势 ${ }^{[1]}$, 与大惯量的机架共同使用时，可组成粗-精跟踪系统， 达到稳定视轴和消除相移的目的, 因而被广泛地应用
在激光通信 ${ }^{[2]}$ 、天文望远镜 ${ }^{[3]}$ 和自适应光学系统的波 前校正 ${ }^{[4]}$ 中。

目前, 对快速反射镜系统的研究主要集中在支承 结构的设计优化 ${ }^{[5]}$ 以及控制系统的高精度高带宽设 计、扰动抑制 ${ }^{[6]}$ 等方面。为了提高音圈电机驱动快速 反射镜的动态响应特性, 中科院长春光机所的王帅 ${ }^{[7]}$ 
提出了一种基于电流环的控制方法, 通过为每个音圈 电机配置一个高带宽的电流环, 实现同一运动轴上的 两个音圈电机的同步推拉作用; 中科院光电所的邓 超、田竟 ${ }^{[8-9]}$ 在分析建立音圈电机驱动的快速反射镜传 递函数模型基础上，设计了基于多种多环路控制的干 扰观测器, 实现对扰动的抑制; 北京航空航天大学的 桂潇怡 ${ }^{[10]}$ 通过在快速反射镜底座中设置两组 MEMS 加速度传感器测量干扰形成的控制前馈, 并采用自适 应方法调节前馈环节系数, 提高了振动环境下系统的 稳定性能。

这些方法在进行系统设计时, 使用的被控对象数 学模型都是频域传递函数模型, 提高控制精度或者进 行干扰抑制时, 均使用了额外的传感器, 多传感器的 使用必然增加硬件系统的复杂性。为了在不增加控制 系统物理硬件复杂性的基础上得到期望的系统稳态 性能和瞬态性能, 同时实现对干扰信号的抑制, 提出 了一种基于系统辨识的状态模型建立方法, 该方法在 建立状态模型的过程中除辨识出的系统参数外仅用 到了音圈电机的线圈电阻和电感, 大大减少了建模过 程中结构参数的使用, 基于状态模型, 设计了由降阶 观测器、状态反馈、内模和镇定补偿器构成的组合系 统, 组合系统在不增加额外传感器的基础上实现了多 环控制的目的, 同时实现了渐进跟踪和干扰抑制的目 标。最后, 对控制系统进行仿真验证, 验证所设计的 控制系统的跟踪性能和扰动抑制能力。

\section{1 快速反射镜的状态模型}

音圈电机驱动的二自由度快速反射镜具有良好 的对称性, 为了便于分析, 对其中的一个运动轴进行 动力学分析, 转矩平衡方程为:

$$
\left(J+2 m_{\mathrm{c}} l^{2}\right) \ddot{\theta}+2 c l^{2} \dot{\theta}+k \theta=P
$$

式中: $J$ 为镜片的转动惯量; $\theta$ 为反射镜的输出转角; $m_{\mathrm{c}}$ 为音圈电机线圈的质量; $c$ 为等效的阻尼系数; $k$ 为柔性支承结构的转动刚度; $l$ 为音圈电机作用点到 转动中心的距离; $P$ 是音圈电机作用在运动方向上的 力矩, 大小为:

$$
P=2 k_{\mathrm{f}} l i
$$

式中: $i$ 为通过音圈电机的电流; $k_{\mathrm{f}}$ 是音圈电机力矩系 数。

音圈电机为反射镜运动机构提供驱动力矩, 根据 基尔霍夫电压定律有

$$
u=L \frac{\mathrm{d} i}{\mathrm{~d} t}+R i+K_{e} \dot{x}
$$

式中: $u$ 是音圈电机的工作电压; $L$ 是音圈电机线圈
的电感; $K_{\mathrm{e}}$ 是音圈电机的反电动势系数; $x$ 为音圈电 机运动的位移。快速反射镜的运动范围较小, 近似有:

$$
x \approx l \theta
$$

联立以上各式，采用古典控制理论的建模方法可 知快速反射镜系统数学模型为一个振荡环节和惯性 环节组成的三阶线性定常系统，其传递函数可表示 为:

$$
\begin{aligned}
G(s)=\frac{\theta(s)}{U(s)} & =\frac{q_{1}}{\left(q_{2} s^{2}+q_{3} s+1\right)\left(q_{4} s+1\right)} \\
& =\frac{\beta_{0}}{s^{3}+a_{2} s^{2}+a_{1} s+a_{0}}
\end{aligned}
$$

式中: $q_{1} 、 q_{2} 、 q_{3} 、 q_{4}$ 是待辨识的快速反射镜参数; $a_{0} 、 a_{1} 、 a_{2} 、 \beta_{0}$ 是常系数。

根据式(1)、式(2)、式(3)和式(4), 选取负载反射 镜的转动角度 $\theta$ 、角速度 $\omega$ 、通过音圈电机的电流 $i$ 为 系统状态变量, 可以得到快速反射镜的状态空间方 程:

$$
\begin{gathered}
\frac{\mathrm{d}}{\mathrm{d} t}\left[\begin{array}{l}
\theta \\
\omega \\
i
\end{array}\right]=\left[\begin{array}{ccc}
0 & 1 & 0 \\
-\frac{k}{J+2 m_{c} l^{2}} & -\frac{2 c l^{2}}{J+2 m_{c} l^{2}} & \frac{2 k_{f} l}{J+2 m_{c} l^{2}} \\
0 & -\frac{k_{e} l}{L} & -\frac{R}{L}
\end{array}\right]\left[\begin{array}{l}
\theta \\
\omega \\
i
\end{array}\right]+\left[\begin{array}{l}
0 \\
0 \\
\frac{1}{L}
\end{array}\right] u \\
y=\left[\begin{array}{lll}
1 & 0 & 0
\end{array}\right]\left[\begin{array}{l}
\theta \\
\omega \\
i
\end{array}\right]
\end{gathered}
$$

联立式(5)、式(6)有:

$$
\left\{\begin{array}{l}
\frac{2 k_{f} l}{J+2 m_{c} l^{2}} \cdot \frac{R}{L}=\beta_{0} \\
\frac{2 c l^{2}}{J+2 m_{c} l^{2}}+\frac{R}{L}=a_{2} \\
\frac{k_{\theta}}{J+2 m_{c} l^{2}}+\frac{2 c l^{2}}{J+2 m_{c} l^{2}} \cdot \frac{R}{L}+\frac{2 k_{f} l}{J+2 m_{c} l^{2}} \cdot \frac{k_{e} l}{L}=a_{1} \\
\frac{k_{\theta}}{J+2 m_{c} l^{2}} \cdot \frac{R}{L}=a_{0}
\end{array}\right.
$$

建立快速反射镜的状态空间模型，方法之一是获 取到快速反射镜的各个参数, 这样状态模型自然可以 随之得到, 但是由于影响快速反射镜结构参数的量有 20 个之多, 其中很多参数是很难甚至是无法得到, 因 此采用这种方法建立快速反射镜的状态模型也变得 很困难。

通过对式(7)进行分析可知, 如果可以通过系统辨 识得到快速反射镜传递函数的参数 $a_{0}, a_{1}, a_{2}, \beta_{0}$, 那么 
确定状态模型时只需要得到音圈电机的电阻 $R$ 和电感

$L$, 使用这 6 个参量即可完成状态空间模型的搭建。

\section{2 控制系统设计}

在经典控制理论中, 提高快速反射镜系统的控制 精度的普遍的做法是将负载反射镜的位置、速度和加 速度或者位置、速度和电流等信号同时进行检测并组 成多环控制系统, 再为每个环路设计合适的控制器达 到提高系统输出精度的目的。工程上, 速度、加速度 以及电流这些物理量虽然是可以测量的, 但是检测装 置价格也都比较昂贵, 这必然会增加控制系统的成本 与增加系统的复杂性。基于快速反射镜的状态模型, 可以有效地解决上述问题, 通过设计内模、补偿器以 及进行状态反馈, 同样可以实现多环控制的效果, 达 到提高控制精度的目的。针对状态反馈过程中难以获 取的物理量, 可以设计降阶观测器, 对状态变量进行 估计, 然后再将其应用到状态反馈环节中。基于状态 模型的组合系统控制框图如图 1 所示, 下面分别对各 环节进行参数设计。

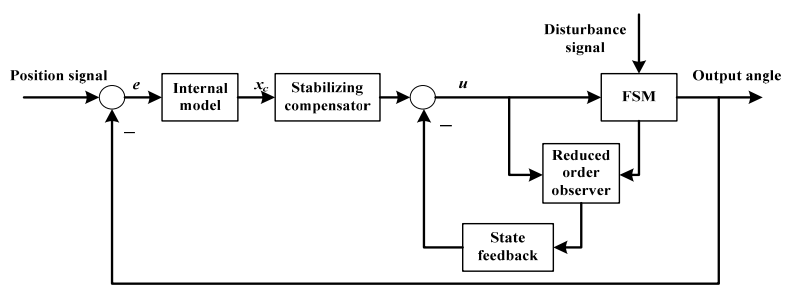

图 1 快速反射镜组合控制系统

Fig.1 Composite control system for fast steering mirror 先将快速反射镜系统方程写成如下形式:

$$
\begin{aligned}
& \dot{\boldsymbol{x}}=\boldsymbol{A} \boldsymbol{x}+\boldsymbol{b} u+\boldsymbol{d}_{f} f(t) \\
& y=\boldsymbol{C} \boldsymbol{x}
\end{aligned}
$$

假定系统能控能观, $\boldsymbol{A} 、 \boldsymbol{b} 、 \boldsymbol{C}$ 分别是对应式(6) 的矩阵, $f(t)$ 是干扰信号且满足状态方程:

$$
\begin{aligned}
& \dot{\boldsymbol{x}}_{f}=\boldsymbol{A}_{f} \boldsymbol{x}_{f} \\
& f(t)=\boldsymbol{C}_{f} \boldsymbol{x}_{f}
\end{aligned}
$$

输入信号 $r(t)$ 由下列方程产生:

$$
\begin{aligned}
& \dot{\boldsymbol{x}}_{r}=\boldsymbol{A}_{r} \boldsymbol{x}_{r}(t) \\
& r(t)=\boldsymbol{C}_{r} \boldsymbol{x}_{r}(t)
\end{aligned}
$$

假定上述干扰信号和输入信号均可测量, $\phi_{f}(s)$ 和 $\phi_{r}(s)$ 分别是干扰信号 $f(t)$ 和输入信号 $r(t)$ 的特征多项 式, 即:

$$
\begin{aligned}
& \varphi_{f}(s)=\operatorname{det}\left(s I-A_{f}\right) \\
& \varphi_{r}(s)=\operatorname{det}\left(s I-A_{r}\right)
\end{aligned}
$$

令 $\phi(s)$ 为 $\phi_{f}(s)$ 和 $\phi_{r}(s)$ 在 $s$ 右半闭平面零点的最小公 倍式，则其展开式可以表示如下:

$$
\varphi(s)=s^{m}+a_{m-1} s^{m-1}+\cdots+a_{1} s+a_{0}
$$

根据内模原理，内模 $\phi^{-1}(s)$ 的状态空间模型可实 现为:

$$
\begin{aligned}
& \dot{\boldsymbol{x}}_{c}=\boldsymbol{A}_{c} \boldsymbol{x}_{c}+\boldsymbol{b}_{c} \boldsymbol{e} \\
& y_{c}=\boldsymbol{x}_{c}
\end{aligned}
$$

式中: $\boldsymbol{A}_{c}=\left[\begin{array}{lrrrr}0 & 1 & 0 & \cdots & 0 \\ 0 & 0 & 1 & \ldots & 0 \\ \vdots & & & \ddots & \\ 0 & 0 & \cdots & & 1 \\ -a_{0} & -a_{1} & \cdots & -a_{m-1}\end{array}\right], \quad \boldsymbol{b}_{c}=\left[\begin{array}{l}0 \\ \vdots \\ 0 \\ 1\end{array}\right]$, 系 统跟踪误差 $e=r-y$ 。

在不考虑干扰信号时, 误差信号 $e=r-C \boldsymbol{x}$, 可得 内模状态方程为:

$$
\dot{\boldsymbol{x}}_{c}=\boldsymbol{A}_{c} \boldsymbol{x}_{c}-\boldsymbol{b}_{c} \boldsymbol{C} \boldsymbol{x}+\boldsymbol{b}_{c} r
$$

快速反射镜系统与内模串联组成的系统状态方 程为:

$$
\left[\begin{array}{l}
\dot{\boldsymbol{x}} \\
\dot{\boldsymbol{x}}_{c}
\end{array}\right]=\left[\begin{array}{ll}
\boldsymbol{A} & 0 \\
-\boldsymbol{b}_{c} C & \boldsymbol{A}_{c}
\end{array}\right]\left[\begin{array}{l}
\boldsymbol{x} \\
\boldsymbol{x}_{c}
\end{array}\right]+\left[\begin{array}{l}
\boldsymbol{b} \\
0
\end{array}\right] u+\left[\begin{array}{l}
0 \\
\boldsymbol{b}_{c}
\end{array}\right] r
$$

假设组合系统能控, 引入状态反馈控制 $K_{\mathrm{c}}$ 和镇 定补偿器 $K$, 控制输出为:

$$
\boldsymbol{u}=\boldsymbol{K}_{c} \boldsymbol{x}_{c}-\boldsymbol{K} \boldsymbol{x}
$$

引入状态反馈后的组合系统的状态方程为:

$$
\left[\begin{array}{c}
\dot{\boldsymbol{x}} \\
\dot{\boldsymbol{x}}_{c}
\end{array}\right]=\left[\begin{array}{ll}
\boldsymbol{A}-\boldsymbol{b} \boldsymbol{K} & \boldsymbol{b} \boldsymbol{K}_{c} \\
-\boldsymbol{b}_{c} \boldsymbol{C} & \boldsymbol{A}_{c}
\end{array}\right]\left[\begin{array}{l}
\boldsymbol{x} \\
\boldsymbol{x}_{c}
\end{array}\right]+\left[\begin{array}{l}
0 \\
\boldsymbol{b}_{c}
\end{array}\right] r
$$

下面继续对受控快速反射镜系统进行研究。系统 输出 $y$ 是反射镜的转角, 将这个状态变量用 $x_{1}$ 表示。 组合系统需要使用的是转角, 角速度和电流 3 个状态 变量, 因为转角已知, 要得到全部 3 个状态变量, 只 需要构造一个低阶的观测器估计剩余的 2 个状态即 可, 这 2 个状态变量用 $x_{2}$ 表示, 据此, 可将受控快速 反射镜系统的状态空间模型写成如下形式:

$$
\begin{aligned}
& \left(\begin{array}{l}
\dot{x}_{1} \\
\dot{\boldsymbol{x}}_{2}
\end{array}\right)=\left(\begin{array}{ll}
\boldsymbol{A}_{11} & \boldsymbol{A}_{12} \\
\boldsymbol{A}_{21} & \boldsymbol{A}_{22}
\end{array}\right)\left(\begin{array}{l}
x_{1} \\
\boldsymbol{x}_{2}
\end{array}\right)+\left(\begin{array}{l}
\boldsymbol{B}_{1} \\
\boldsymbol{B}_{2}
\end{array}\right) u \\
& y=\left[\begin{array}{ll}
I & 0
\end{array}\right]\left(\begin{array}{l}
x_{1} \\
\boldsymbol{x}_{2}
\end{array}\right)=x_{1}
\end{aligned}
$$

对上式进行展开，有:

$$
\begin{aligned}
& \dot{x}_{1}=\boldsymbol{A}_{11} x_{1}+\boldsymbol{A}_{12} \boldsymbol{x}_{2}+\boldsymbol{B}_{1} u \\
& \dot{\boldsymbol{x}}_{2}=\boldsymbol{A}_{21} x_{1}+\boldsymbol{A}_{22} \boldsymbol{x}_{2}+\boldsymbol{B}_{2} u \\
& y=x_{1}
\end{aligned}
$$

下面对不能测量得到的电流和角速度两个状态 
变量设计观测器进行观测, 令:

$$
\begin{aligned}
& \boldsymbol{M}=\boldsymbol{A}_{21} x_{1}+\boldsymbol{B}_{2} u \\
& \boldsymbol{Z}=\dot{x}_{1}-\boldsymbol{A}_{11} x_{1}-\boldsymbol{B}_{1} u=\boldsymbol{A}_{12} \boldsymbol{x}_{2}
\end{aligned}
$$

则上式可以变成:

$$
\begin{aligned}
& \dot{\boldsymbol{x}}_{2}=\boldsymbol{A}_{22} \boldsymbol{x}_{2}+\boldsymbol{M} \\
& \boldsymbol{Z}=\boldsymbol{A}_{12} \boldsymbol{x}_{2}
\end{aligned}
$$

如果将 $M$ 视为系统输入量, $Z$ 视为输出量, $A_{22}$ 和 $A_{12}$ 分别视作系统矩阵和输出矩阵, 则上式可以看 成是受控快速反射镜系统的一个子系统, 2 个状态变 量均不能直接量测得到, 为此可以通过构建一个 2 维 的状态观测器, 实现对这 2 个状态的重构, 状态不可 量测子系统的状态观测器为:

$$
\dot{\hat{\boldsymbol{x}}}_{2}=\left(\boldsymbol{A}_{22}-\boldsymbol{G} \boldsymbol{A}_{12}\right) \hat{\boldsymbol{x}}_{2}+\boldsymbol{M}+\boldsymbol{G} \boldsymbol{Z}
$$

为了在原系统中可以实现该状态观测器, 需要消 去中间变量 $\boldsymbol{M}, \boldsymbol{Z}$ ，将式(20)带入式(22), 可得：

$$
\dot{\hat{\boldsymbol{x}}}_{2}=\left(\boldsymbol{A}_{22}-\boldsymbol{G} \boldsymbol{A}_{12}\right) \hat{\boldsymbol{x}}_{2}+\left(\boldsymbol{A}_{21} y+\boldsymbol{B}_{2} u\right)+\boldsymbol{G}\left(\dot{y}-\boldsymbol{A}_{11} y-\boldsymbol{B}_{1} u\right)
$$

工程中的信号通常包含有大量的噪声, 在系统中 直接对输出信号进行微分会在控制系统引入噪声干 扰, 为避免微分运算, 引入中间变量 $\omega$, 令:

$$
\omega=\hat{x}_{2}-G y
$$

对变量 $\omega$ 进行求导, 经整理可得:

$$
\begin{aligned}
\dot{\boldsymbol{\omega}}= & \left(\boldsymbol{A}_{22}-\boldsymbol{G} \boldsymbol{A}_{12}\right) \omega+\left[\left(\boldsymbol{A}_{22}-\boldsymbol{G} \boldsymbol{A}_{12}\right) \boldsymbol{G}+\boldsymbol{A}_{21}-\boldsymbol{G} \boldsymbol{A}_{11}\right] y+ \\
& \left(\boldsymbol{B}_{2}-\boldsymbol{G} \boldsymbol{B}_{1}\right) u
\end{aligned}
$$

降阶观测器得到系统状态变量为:

$$
\hat{\boldsymbol{x}}_{2}=\boldsymbol{w}+\boldsymbol{G} \boldsymbol{y}
$$

系统状态 $\hat{\boldsymbol{x}}=\left[\begin{array}{l}\boldsymbol{x}_{1} \\ \hat{\boldsymbol{x}}_{2}\end{array}\right]$, 加入降阶观测器的组合系统 的状态方程为:

$$
\left[\begin{array}{l}
\dot{\hat{\boldsymbol{x}}} \\
\dot{\boldsymbol{x}}_{c}
\end{array}\right]=\left[\begin{array}{ll}
\boldsymbol{A}-\boldsymbol{b} \boldsymbol{K} & \boldsymbol{b} \boldsymbol{K}_{\boldsymbol{c}} \\
-\boldsymbol{b}_{c} \boldsymbol{C} & \boldsymbol{A}_{c}
\end{array}\right]\left[\begin{array}{l}
\hat{\boldsymbol{x}} \\
\boldsymbol{x}_{c}
\end{array}\right]+\left[\begin{array}{l}
0 \\
\boldsymbol{b}_{c}
\end{array}\right] \boldsymbol{r}
$$

根据快速反射镜控制系统的性能指标要求, 确定 出控制系统闭环主导极点的位置, 保证另一极点的实 部比主导极点的实部大 $3 \sim 6$ 倍以上时可忽略该极点 对控制系统输出性能的影响。降阶观测器的特征根应 该有比状态反馈系统特征根更大的实部模。

由闭环系统主导极点确定的特征方程式和组合 系统的特征方程式相等, 即可确定反馈环节和镇定补
偿器的参数。

\section{3 仿真实验与结果分析}

对一款工程中使用的快速反射镜进行了系统辨 识, 通过拟合得到的快速反射镜开环模型为:

$$
\tilde{G}_{\mathrm{FSM}}(s)=\frac{0.6012}{0.00001057 s^{2}+0.00065225 s+1} \cdot \frac{1}{0.00053 s+1}
$$

音圈电机的标称电感和电阻分别为 $1.9 \mathrm{mH}$ 和 3.6

$\Omega$, 得到快速反射镜的状态方程为:

$$
\begin{gathered}
{\left[\begin{array}{c}
\dot{\theta} \\
\ddot{\theta} \\
\dot{i}
\end{array}\right]=\left[\begin{array}{lcc}
0 & 1 & 0 \\
-94303 & -52 & 203680 \\
0 & -0.08 & -1895
\end{array}\right]\left[\begin{array}{l}
\theta \\
\dot{\theta} \\
i
\end{array}\right]+\left[\begin{array}{l}
0 \\
0 \\
526
\end{array}\right] u} \\
y=\theta
\end{gathered}
$$

采用扫频法辨识出的快速反射镜开环系统模型、 拟合得到的系统模型以及状态模型幅频特性曲线对 比图如图 2 所示, 从图中可以看到状态模型与辨识出 的系统曲线基本重合。

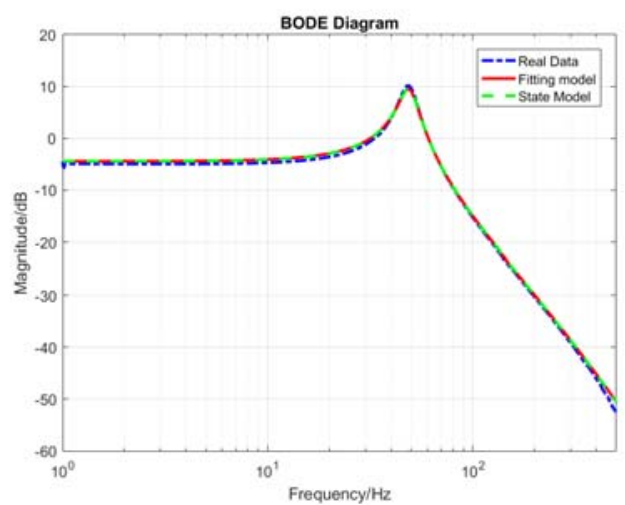

图 2 幅频特性曲线对比图

Fig.2 Comparison diagram of amplitude-frequency curves

对于系统来说，阶跃信号是最具挑战性的，故选 择单位阶跃信号 $r(t)=1(t)$ 作为系统测试输入, 扰动信 号选为正弦信号 $f(t)=\sin (t)$, 可得 $\phi(s)=s^{3}+s$, 内模可 实现为:

$$
\begin{aligned}
& \dot{\boldsymbol{x}}_{c}=\left[\begin{array}{rrr}
0 & 1 & 0 \\
0 & 0 & 1 \\
0 & -1 & 0
\end{array}\right] \boldsymbol{x}_{c}+\left[\begin{array}{l}
0 \\
0 \\
1
\end{array}\right] \mathrm{e} \\
& \boldsymbol{y}_{c}=\boldsymbol{x}_{c}
\end{aligned}
$$

给定快速反射镜闭环控制系统的设计指标是：超 调量小于 $10 \%$ ，调节时间小于 $10 \mathrm{~ms}$ 。通过配置状态 反馈环节的参数将系统的极点配置在复平面希望位 置上，结合主导极点的定义，可将系统的预期的主导 
极点取为 $S_{1,2}=-440 \pm \mathrm{j} 448$, 其他极点为:

$$
S_{3,4}=-2000 \pm \mathrm{j} 1000, S_{4,5}=-3000 \pm \mathrm{j} 1500
$$

计算并对参数修正后得状态反馈环节和镇定补 偿器分别为:

$$
\begin{gathered}
K=[1057.9,0.4552,16.9829] \\
K_{\mathrm{c}}=\left[2.2303,7.3806 \times 10^{6}, 1.2892 \times 10^{6}\right]
\end{gathered}
$$

取降阶状态观测器的特征值为: $s_{1}=-40000$, $s_{2}=-45000$, 则降阶观测器状态观测矩阵 $\boldsymbol{G}=\left[\begin{array}{l}8.305 \times 10^{4} \\ 8.064 \times 10^{3}\end{array}\right]$, 到此, 组合控制系统的各环节的参 数设计完成。

由于采用改进根轨迹的方法设计不完全微分 PID 控制系统的参数时, 也是以时域性能指标要求为设计 出发点, 因此选择以同样性能指标要求时采用该种方 法确定的控制系统作为对照实验系统。不考虑扰动信 号作用时, 两种方法的系统输出曲线如图 3 所示。从 图中可以得到, 不完全微分 PID 控制系统的峰值时间 为 $0.0070 \mathrm{~s}$, 调节时间为 $0.0049 \mathrm{~s}$, 超调量为 $4.0195 \%$, 而组合系统的峰值时间为 $0.0019 \mathrm{~s}$, 调节时间为 0.0023 $\mathrm{s}$, 超调量为 $9.2950 \%$, 相较于不完全微分 PID 控制系 统, 组合系统的峰值时间下降了 $72.85 \%$, 调节时间下 降了 $53.06 \%$, 超调量则上升了 $131.2 \%$, 组合系统的 超调量虽有所上升，但仍然满足性能指标的要求。

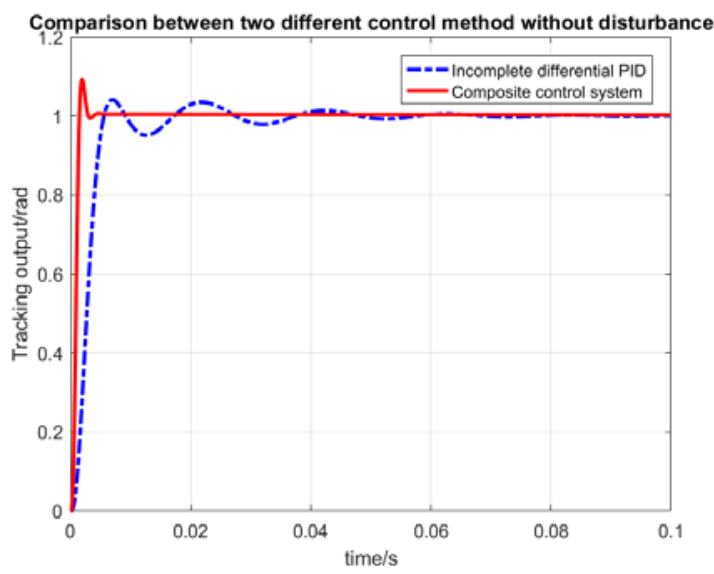

图 3 不考虑干扰时两种控制方法输出曲线

Fig.3 Output curves of two control method without considering disturbance

在系统中施加幅值为 1 , 圆频率为 $100 \mathrm{rad} / \mathrm{s}$ 的正 弦干扰信号前后不完全微分 PID控制系统的输出曲线 如图 4 所示。从图中可以看到, 施加扰动作用后系统 输出会在平衡点上下震荡, 影响了控制系统的稳态精 度。

加入同样的扰动后, 组合系统的输出曲线如图 5 所示, 从图中可以看出, 加入干扰信号作用后组合系
统的峰值时间为 $0.0019 \mathrm{~s}$, 调节时间为 $0.0023 \mathrm{~s}$, 超调 量为 $9.2950 \%$, 加入干扰信号前后, 组合系统的输出 曲线基本重合，峰值时间、调节时间和超调量等时域 性能指标也完全一样。仿真结果证明了基于状态反馈 的组合系统可以同时实现对输入的渐进跟踪和对干 扰信号的抑制。

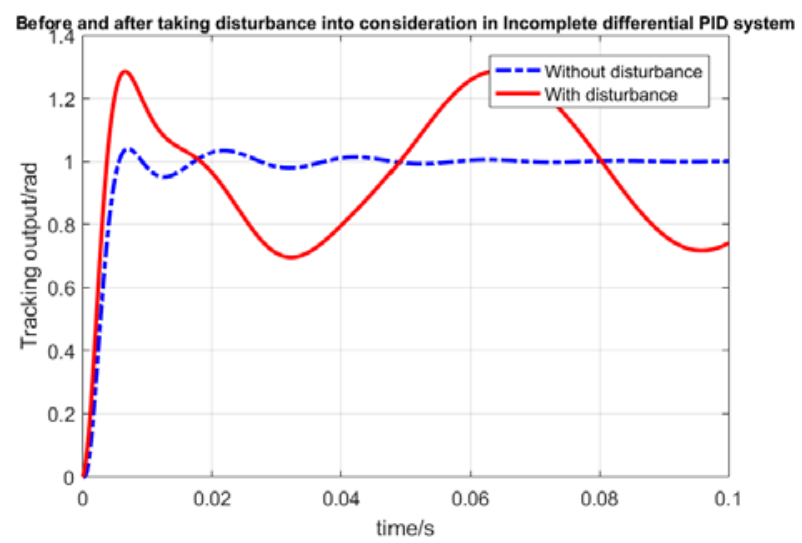

图 4 幅值为 1 频率为 $100 \mathrm{rad} / \mathrm{s}$ 的干扰作用前后不完全微分系 统输出曲线对比

Fig.4 Comparison of output curves at amplitude 1 and frequency $100 \mathrm{rad} / \mathrm{s}$ before and after taking disturbance into consideration in incomplete differential PID system

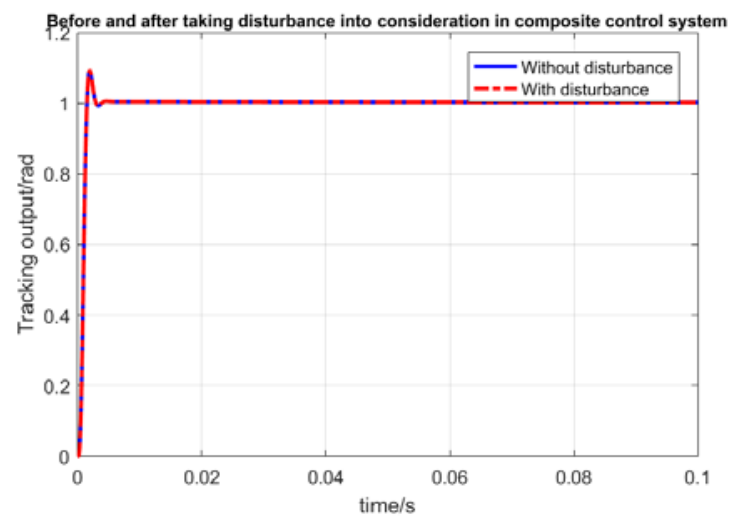

图 5 幅值为 1 频率为 $100 \mathrm{rad} / \mathrm{s}$ 的干扰作用前后组合系统输出 曲线对比

Fig.5 Comparison of output curves at amplitude 1 and frequency $100 \mathrm{rad} / \mathrm{s}$ before and after taking disturbance into consideration in composite system

加大干扰信号作用, 在系统中施加幅值为 10 , 圆 频率为 $1000 \mathrm{rad} / \mathrm{s}$ 的正弦干扰信号, 加入干扰信号前 后, 不完全微分 PID 控制系统的输出曲线和组合系统 的输出曲线分别如图 6 和图 7 所示。从图 6 中可以看 到, 加大干扰信号作用后, 不完全微分 PID 控制系统 的输出完全失真; 从图 7 中可以看到, 加大干扰信号 作用后, 组合系统的峰值时间为 $0.0019 \mathrm{~s}$, 调节时间 为 $0.0023 \mathrm{~s}$, 超调量为 $9.4326 \%$, 可以发现组合系统 
受干扰信号的影响很小。通过对比图 5 和图 7 还可以 发现组合系统对扰动信号的频率也具有较低的灵敏 度, 这是由于在进行组合系统设计过程中用内模抵消 了干扰信号的作用, 实现了组合系统对扰动的全频段 抑制, 与理论期待相符。

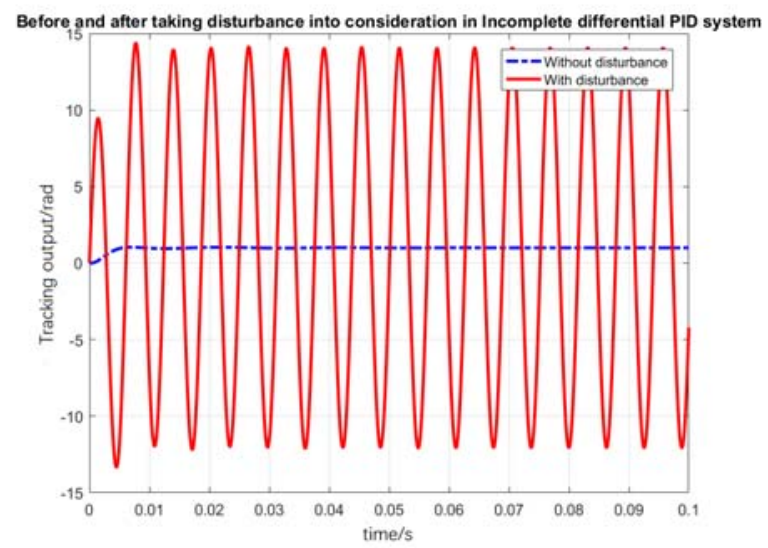

图 6 幅值为 10 频率为 $1000 \mathrm{rad} / \mathrm{s}$ 的干扰作用前后不完全微分 系统输出曲线对比

Fig.6 Comparison of output curves at amplitude 10 and frequency $1000 \mathrm{rad} / \mathrm{s}$ before and after taking disturbance into consideration in incomplete differential PID system

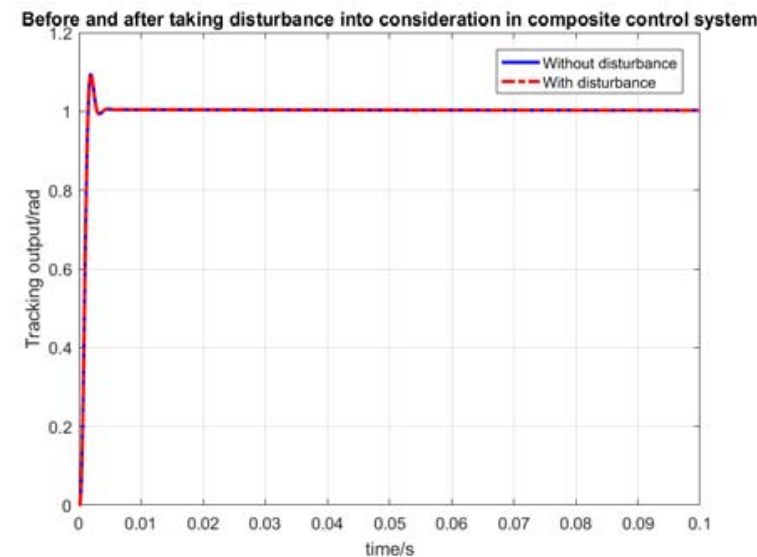

图 7 幅值为 10 频率为 $1000 \mathrm{rad} / \mathrm{s}$ 的干扰作用前后组合系统输 出曲线对比

Fig.7 Comparison of output curves at amplitude 10 and frequency $1000 \mathrm{rad} / \mathrm{s}$ before and after taking disturbance into consideration in composite system

\section{4 结论}

为使得设计的控制系统可以同时实现对输入信 号的渐进跟踪和对可测干扰信号的抑制, 亦不增加控 制系统硬件复杂程度, 本文从状态模型的快速建立为 出发点, 提出一种基于系统辨识的状态模型建立方 法, 该方法只需要使用 6 个参量即可完成状态模型的 建立, 大大减少了建模过程中结构参数的使用。基于
快速反射镜的状态模型, 设计了一套组合控制系统, 并着重分析了组合系统中内模、状态反馈和镇定补偿 器的设计方法, 给出了组合系统中降阶观测器实现方 法, 分析计算出组合系统状态方程。对工程上使用的 一款快速反射镜应用了上述组合控制系统, 给出了控 制系统的具体参数, 并进行了仿真验证, 仿真结果表 明理论分析的正确性。

\section{参考文献:}

[1] Kluk D J, Boulet M T, Trumper D L. A high-bandwidth, high-precision, two-axis steering mirror with moving iron actuator[J]. Mechatronics, 2012, 22: 257-270.

[2] 赵否, 纪明, 赵振海, 等. 舰载激光武器稳定平台粗精复合控制 [J]. 激光与红外, 2019, 49(1): 86-92.

ZHAO Lei, JI Ming, ZHAO Zhenhai, et, al. Primary-precise compounded control for stabilized platform in shipborne laser weapon[J]. Laser and Infrared, 2019, 49(1): 86-92.

[3] 吕世良, 刘金国, 周怀得, 等. 星载红外探测器快速反射镜控制系统 设计[J]. 红外与激光工程, 2017, 46(9): 102-107.

LYU Shiliang, LIU Jinguo, ZHOU Huaide, et al. Design of control system for fast steering mirror of infrared detector based on satellite[J]. Infrared and Laser Engineering, 2017, 46(9): 102-107.

[4] WANG Kaidi, SU Xiuqin, LI Zhe, et al. Time-frequency characteristics optimal control of fast steering mirror for image motion compensation[J]. Infrared and Laser Engineering, 2018, 47(S1): 120-126.

[5] 鲁亚飞, 范大鹏, 范世珣, 等. 快速反射镜两轴柔性支承设计 [J]. 光 学精密工程, 2010, 18(12): 2574-2582.

LU Yafei, FAN Dapeng, FAN Shixun, et, al. Design of two-axis elastic support for fast steering mirror[J]. Optics and Precision Engineering, 2010, 18(12): 2574-2582.

[6] 彭树萍, 于洪君, 王伟国, 等. 新型快速反射镜伺服系统设计 $[\mathrm{J}]$. 红 外与激光工程, 2014, 43(5): 1610-1615.

PENG Shuping, YU Hongjun, WANG Weiguo, et al. Design of servo system for novel fast-steering mirror[J]. Infrared and Laser Engineering, 2014, 43(5): 1610-1615.

[7] 王帅, 曹玉岩. 音圈电机型快速反射镜的驱动控制系统设计 [J]. 电 子测量与仪器学报, 2017, 31(7): 1115-1121.

WANG Shuai, CAO Yuyan. Design of control system for fast steering mirror driven by voice coil actuator[J]. Journal of Electronic Measurement and Instrumentation, 2017, 31(7): 1115 - 1121.

[8] DENG Chao, TANG Tao, MAO Yao, et al. Enhanced disturbance observer based on acceleration measurement for fast steering mirror system[J]. IEEE Photonics Journal, 2017, 9(3): 1-11.

[9] TIAN Jing, YANG Wenshu, PENG Zhenming. Application of MEMS Accelerometers and Gyroscopes in Fast Steering Mirror Control Systems[J]. Sensors, 2016, 440: 1-13.

[10] 桂潇怡, 李伟鹏, 郑新涛, 等. 基于 MEMS 加速度计的快速反射镜 复合控制[J]. 航天控制, 2018, 36(2): 30-36, 41.

GUI Xiaoyi, LI Weipeng, ZHENG Xintao, et al. Composite Control of Fast Steering Mirror Based on MEMS Accelerometer[J]. Aerospace Control, 2018, 36(2): 30-36, 41. 